\title{
Paul-Emmanuel Stradda, Essai sur l'existence de Dieu. Revue Metaphysica theoria - Tome 6
}

Paris, L'Harmattan, coll. « Ouverture philosophique », 2012, 288 p., Illustrations.

\section{Mustapha Naïmi}

\section{(2) OpenEdition} Journals

Édition électronique

URL : http://journals.openedition.org/assr/26577

DOI : $10.4000 /$ assr.26577

ISSN : $1777-5825$

Éditeur

Éditions de l'EHESS

Édition imprimée

Date de publication : 31 décembre 2014

Pagination : 298

ISBN : 978-2-7132-2467-6

ISSN : 0335-5985

Référence électronique

Mustapha Naïmi, «Paul-Emmanuel Stradda, Essai sur l'existence de Dieu. Revue Metaphysica theoria - Tome 6 ", Archives de sciences sociales des religions [En ligne], 168|2014, mis en ligne le 21 mai 2015, consulté le 22 septembre 2020. URL : http://journals.openedition.org/assr/26577 ; DOI : https:// doi.org/10.4000/assr.26577

Ce document a été généré automatiquement le 22 septembre 2020

(C) Archives de sciences sociales des religions 


\section{Paul-Emmanuel Stradda, Essai sur l'existence de Dieu. Revue Metaphysica theoria - Tome 6}

Paris, L'Harmattan, coll. « Ouverture philosophique », 2012, 288 p., Illustrations.

Mustapha Naïmi

\section{RÉFÉRENCE}

Paul-Emmanuel Stradda, Essai sur l'existence de Dieu. Revue Metaphysica theoria -

Tome 6, Paris, L'Harmattan, coll. « Ouverture philosophique », 2012, 288 p.

1 Ce sixième tome de la collection "Ouverture philosophique " traite, sur un plan méthodologique destiné sous la forme de manuel aux étudiants, de trois parties: la première tente de définir la nécessité ontologique et métaphysique de l'être et l'unité ; la seconde partie évoque la nécessité spirituelle et religieuse de Dieu selon un lien établi entre l'être et la relation; la troisième partie traite de la nécessité éthique et axiologique de l'homme (l'être et l'amour).

2 La thèse transcendante de Dieu passe par l'intuition supra conceptuelle d'un au-delà de l'être. On retrouve cette opinion dans la métaphysique zen ou new age. Elle est développée par René Guénon qui, pour définir la métaphysique, explique qu'il faut partir de l'étymologie: "D'après sa composition, le mot "métaphysique" signifie littéralement au-delà de la "physique". Or, la "physique" est l'étude de tout ce qui appartient au domaine de la nature; ce qui concerne la métaphysique, c'est ce qui est au-delà de la nature ». D'où la conséquence que Guénon s'empresse de tirer: la connaissance métaphysique est une connaissance non pas naturelle mais surnaturelle. Son objet est au-delà de l'être; toute détermination est conçue comme une limite et une aliénation dont il faut se délivrer. La métaphysique est au-delà de toutes nos formules et de nos systèmes. 
3 L'esprit interroge l'être, est saisi par lui, et porte une connaissance sur l'être. Dans cette perspective, l'être offre à l'esprit qui le saisit la possibilité de trouver en lui une lecture quasi immédiate de l'existence de Dieu. Alors l'esprit qui saisit l'être est saisi lui-même par sa propre contingence et par la présence d'une norme transcendante qui le renvoie à Dieu : l'esprit qui saisit est à son tour saisi par la vérité intérieure. Cette vérité intérieure à l'esprit est par excellence le signe révélateur de la présence de Dieu. Nous savons du moins que l'esprit est une relation intrinsèque à l'être, et par-là à l'absolu de l'être qu'est Dieu pour l'être et dans l'esprit. Connaître Dieu, c'est porter une connaissance sur l'être, l'être relationnel; c'est aussi se savoir esprit, et esprit relationnel. Dieu est la relation absolue. Aussi, les constructions de la pensée moderne semblent se « désontologiser " par une dévaluation de l'être et de l'intelligible. Au lieu d'être la voie royale vers les plus hautes vérités. L'atmosphère actuelle de la philosophie se détourne de l'être, qu'elle juge opaque, abstrait ou chimérique à l'esprit.

4 La thèse cosmologique, celle de la révélation cosmique est traitée ensuite à partir des aspects philosophiques et théologiques. Le réel ne portant pas sur la mise en place des éléments, porte sur la totalité de l'être de ce qui est. Le réel est unique, intelligible, ontologique, existentiel. Il est relation de tout ce qui est à son principe. Ce rapport est un Absolu de valeur, philosophique et religieux. En tant qu'il constitue un tout spatiotemporel, l'univers pose la question de l'essentialité de l'espace à la matière. Le temps étant une forme intelligible de l'être, est intérieur à lui et non pas l'être intérieur au temps. Il résulte que l'espace et le temps sont intimement liés et constituent les matériaux de base de l'être fini. Dans la théorie du Big Bang, il s'est produit un commencement, un "moment ", un point de départ. Simultanément et de manière instantanée, à partir du néant, ont été créés l'espace, le temps, et aussi la matérialité de l'univers tout entier. Cet acte constitue le «Fiat Lux» (Que la lumière soit), le «Fiat Cosmos ». Dieu qui est principe (Il dit et cela s'accomplit), nous fait passer du néant à l'être. L'expression " point-univers » ne désigne pas un objet isolé dans l'espace, mais le commencement des temps, le futur univers en attente.

5 Ainsi, la dualité de l'espace et de la matière nous permet de comprendre l'essence de la réalité objective, matérielle. Ontologiquement parlant, disons que la matière est l'étant, dont l'espace est l'être. L'espace permet à la matière d'être telle qu'elle est. L'espace est l'être de la matière, comme la matière est l'étant de l'espace.

6 Ainsi, la matière sans l'espace serait seulement une essence qui ne surgit pas, ne naît pas, n'introduit pas le monde et ne forme pas avec l'espace et le temps ce monde. Le temps et l'espace sont des paramètres interdépendants et relationnels, liés à la matière, formant un "espace-temps » courbé par la matière qu'il contient et qui est peut-être fini. Il est à remarquer que le cosmos serait éternel si le temps n'existait pas. Le cosmos n'aurait pas de commencement. L'exclusion du temps ferait disparaître la réalité de l'espace. Paradoxalement, un cosmos éternel serait le néant.

7 Que serait l'espace? Il n'est pas le temps déchu comme le pensait Heidegger. Du point de vue métaphysique, l'espace n'est pas une forme subjective (au sens kantien), l'espace « est » dans la mesure où il permet à la matière d'être matière. Il ne lui donne pas l'essence, car la matière est en elle-même, mais il permet de se manifester, comme un être matériel achevé dans ce monde matériel. Ainsi donc, l'espace ne donne pas l'essence à la matière car celle-ci est nécessairement ce qu'elle est, mais l'espace permet à la matière d'utiliser une faculté qui lui est propre : être pleinement. À cause 
de cette relation intime et profonde entre la matière et l'espace, sans la matière, nous ne pouvons dire que l'espace n'existe pas.

Ainsi, d'un point de vue métaphysique, l'univers est un tout relationnel avec une unité et une essence. Il constitue un Tout dont chaque fragment suppose l'autre et réciproquement, ce sont là les harmonies qui existent entre les différentes parties de l'univers.

9 Une réalité absolue s'impose, un effectif existe, un absolu existe. Deux éléments sont donc indispensables à cette réflexion de caractère exclusivement métaphysique. La relativité ou l'effectibilité du fini comme tel et la nécessité d'un effectif, d'un absolu. L'origine de l'être remonte à un point précis dans le temps. C'est le point ontologique. L'être du temps est sans doute l'expérience la plus universelle qui soit. Le temps et l'espace sont également liés à l'existence de la matière. La matière est un mélange d'espace et de temps, c'est la matière spatialisée. Le temps est mouvement, variation, croissance ; il est mouvement vers l'être.

10 Il y a deux réalités fondamentales : le temps et la forme. La réalité est forme. La forme est réalité ou être. Ainsi si le temps participe à la forme et que la forme, à son tour, participe finalement à l'être, on conçoit aisément que le temps, lui aussi, participe à l'être. C'est la temporalité et le « finitif » de l'être.

11 De façon philosophique, le principe de causalité renvoie au principe de l'être. Le rapport causalité-être constitue le fondement de la métaphysique ontologique. On peut définir le principe de causalité : tout être fini, conditionné, composé, imparfait et relatif trahit une indigence, une dépendance ou une relativité totale vis-à-vis d'une réalité qui transcende le fini, c'est-à-dire vis-à-vis d'une réalité non finie, immuable, absolument simple, parfaite, intelligente.

12 Concernant la métaphysique et le problème de Dieu, l'auteur pense qu'il n'est pas nécessaire de voir, à côté de l'œuvre d'art, la photographie de l'artiste pour croire à son existence. Le chef-d'œuvre témoigne pour lui. Le créateur de l'univers a donné des preuves en suffisance pour se faire reconnaître partout ce que nos yeux peuvent admirer. Du plus minuscule jusqu'au plus grandiose, tout proclame la sagesse qui a présidé à l'harmonie de cette création. Si, par exemple, on entre dans une usine entièrement automatisée et qu'on n'y voit aucun homme, on n'imaginera pas, et cela sans aucune réserve, que cette usine puisse fonctionner sans un mécanicien qui ait pensé l'organisation des machines et des pièces qui la composent, dont l'esprit relie et met ces machines entre elles et par rapport à l'ordre central. Au contraire même, plus les interventions visibles de l'homme seront rares, plus l'automatisation sera poussée, plus grand apparaîtra le génie du mécanicien. 